\title{
Aphorismen aus der Geometrie des Raumes.
}

(Von Herrn Prof. Dr. Plicker in Bonn.)

\section{Die Axen der Flächen zweiter Ordnung.}

5. 1.

1. Die Absicht dieses Paragraphs ist, auf möglichst einfáche Weise eine für Anwendungen wichtige Aufgabe zu behandeln, welche früher schon verschiedeue Lösungen gefunden hat: die Aufgabe nemlich, der Lage und Gröfse nach, die Axen der Flächen zweiter Ordnung zu bestimmen, wenn diese Flächen durch ihre allgemeine Gleichung zwischen rechtwinkligen Coordinaten gegeben sind. Wir setzen hierbei voraus, dafs die Fläche einen Mittelpunct habe und wollen diesen Mittelpunct zum Anfangspuncte der Coordinaten nehmen. Dann können wir der allgemeinen Gleichung die folgende Form geben :

1. $A x^{2}+A^{\prime} y^{2}+A^{\prime \prime} z^{2}+2 B y z+2 B^{\prime} x z+2 B^{\prime \prime} x y=C$.

Wenn $C$ verschwindet, so stellt diese Gleichung insbesondere Kegelflächen dar; ,abstrahiren wir von diesen, so können wir, unbeschadet der Allgemeinheit, $C=1$ nehmen.

2. Wenn wir, nach beliebiger Richtung die Fläcle durch parallele Ebenen schneiden, so liegen die Mittelpuncte der Durchschnittscurven in allen diesen Ebenen auf einem Durchmesser der Fläche; und wenn dieser Durchmesser auf den Schnitt-Ebenen senkrecht steht, so ist er eine Axe der Fläche. Um die Richtung einer solchen Axe zu finden, brauchen wir also blofs eine', übrigens beliebige Schnitt-Ebene so zu führen, dafs ein vom Mittelpuncte der Fläche auf diese Ebene gefälltes Perpendikel den Mittelpunct der Durchschnittseurve trifft.

Die Gleichung einer solchen Schnitt-Ebene sei

$$
\text { 2. } z+b y+a x=c \text {; }
$$

die Gleichungen des Perpendikels sind alsdann:

$$
\text { 3. } x=a z, \quad y=b z \text {. }
$$


Wir wollen die analytischen -Bedingungen entwickeln, unter welchen dieses Perpendikel den Mittelpunct der Durchschnittscurve in der Ebene (2.) trifft. Um zuvörderst diesen Mittelpunct zu bestimmen, brauchen wir blofs die Gleichung der Fläche (1.) einmal in Beziehung auf $x$, das andere Mal in Beziehung auf $y$ zu differentiiren und hierbei, in Folge der Gleichung (2.), $z$ als Function von $x$ und $y$ zu betrachten, so dafs

$$
\frac{d z}{d x}=-a, \quad \frac{d z}{d y}=-b
$$

Auf diese Weise ergeben sich unmittelbar die folgenden beiden Gleichungen :

$$
\begin{aligned}
\left(\boldsymbol{A} x+\boldsymbol{B}^{\prime \prime} y+\boldsymbol{B}^{\prime} \boldsymbol{z}\right)-\left(\boldsymbol{B}^{\prime} x+\boldsymbol{B} y+\boldsymbol{A}^{\prime \prime} \boldsymbol{z}\right) \boldsymbol{a} & =0 \\
\left(\boldsymbol{B}^{\prime \prime} x+\boldsymbol{A}^{\prime} y+\boldsymbol{B}_{z}\right)-\left(\boldsymbol{B}^{\prime} x+\boldsymbol{B} y+\boldsymbol{A}^{\prime \prime} \boldsymbol{z}\right) \boldsymbol{b} & =0,
\end{aligned}
$$

welche eine gerade Linie darstellen, die die Ebene (2.) in dem gesuchten Mittelpuncte schneidet. *) Mit ihnen zugleich müssen also die beiden Gleichungen (3.) bestehen, wenn die bezügliche gerade Linie eine Axe der Fläche sein soll. Eliminiren wir hiernach zwischen diesen vier Gleichungen $\frac{x}{z}$ und $\frac{y}{z}$, so kommt:

$$
\text { 4. }\left\{\begin{aligned}
\left(\boldsymbol{A} a+\boldsymbol{B}^{\prime \prime} b+\boldsymbol{B}^{\prime}\right)-\left(\boldsymbol{B}^{\prime} a+B b+\boldsymbol{A}^{\prime \prime}\right) a & =0, \\
\left(\boldsymbol{B}^{\prime \prime} a+\boldsymbol{A}^{\prime} b+\boldsymbol{B}\right)-\left(\boldsymbol{B}^{\prime} a+B b+\boldsymbol{A}^{\prime \prime}\right) b & =0 .
\end{aligned}\right.
$$

Durch diese beiden Gleichungen, welche drei Werthenpaare für $b$ und $a$ geben, sind die Richtungen der drei Axen gegeben. Vor diesen Richtungen ist eine offenbar reell, und es ist leicht zu zeigen, dafs die beiden andern es ebenfalls sind. Wenn wir zu diesem Ende annehmen, dafs die notorisch reelle Axenrichtung mit der Axe der $z$ zusammenfalle, so müssen die beiden Gléichungen (4.) befriedigt werden, wenn $a$ und $b$ zugleich verschwinden. Dies fordert $\dot{B}=0$ und $\boldsymbol{B}^{\prime}=0$. Dann reduciren sich aber gleichzeitig diese beiden Gleichungen auf den ersten Grad; was anzeigt, dafs ein zweites Werthenpar von $a$ und $b$ unendlich wird (wir erkennen auch unmittelbar, dafs die Gleichungen (4.) befriedigt werden, wenn $\frac{1}{a}=0$ und $\frac{1}{b}=0$ ist), und dafs also eine zweite Axe in die Ebene $x y$ fällt. Die auf den ersten

*) Wenn wir zwischen den Gleichungen (1.) und (2.) $z$ eliminiren, so erhalten wir die Projection der Durchschnittscurve auf die Ebene $x y$. Um das $x$ und $y$ des Mittelpunctes dieser Projection und also auch der Durchschnittscurve selbst zu finden, brauchen wir bekanntlich nur die resultirende Gleichung nach einander in Beziehung auf $x$ und $y$ zu differentiiren. Wir können hierbei auch wie im Texte verfahren und, uach der Differentiation, aus den Gleichungen (4.), vermittelst (2.), z eliminiren. 
Grad reducirten Gleichungen (4.) werden aber nur befriedigt, wenn $a$. und $b$ beide unendlich grofs werden, oder wenn beide verschwinden. Die dritte Axe kann hiernach also nur entweder ebenfalls in der Ebene $x y$ liegen, oder mit der Axe der z zusammenfallen. Den letztern Fall können wir sogleich, als unstatthaft, ausschliefsen. Um hiernach die Richtung der beiden in der Ebene $x y$ liegenden Axen zu bestimmen, brauchen wir nur aus den beiden Gleichungen (4.) die folgende abzuleiten:

$$
\left(A \boldsymbol{a}+\boldsymbol{B}^{\prime \prime} b+B^{\prime}\right) b-\left(B^{\prime \prime} a+A^{\prime} b+B\right) a=0,
$$

welche durch das Verschwinden vop $B$ und $B^{\prime}$ auf die Gleichung

$$
\text { 5. } B^{\prime \prime}\left(\frac{b}{a}\right)^{2}-\left(A^{\prime}-A\right) \frac{b}{a}-B^{\prime \prime}=0
$$

sich reducirt und anzeigt, dafs diese beiden Axen reell sind und auf einander senkrecht stehen.

Jede Fläche zweiter Ordnung mit einem Mittelpuncte hat also drei, paarweise genommen; auf einander senkrechte, reelle Axen.

3. Nachdem wir die Richtungen der drei Axen gefunden haben, ist es leicht, ihre Grölse zu bestimmen. Für den Durchschnittspunct einer Axe und der Fläche bestehen gleichzeitig die drei Gleichungen (1.) und (3.). Hieraus ergiebt sich, wenn wir zugleich $G=1$ setzen :

$$
\left(A a^{2}+A^{\prime} b^{2}+A^{\prime \prime}+2 B b+2 B^{\prime} a+2 B^{\prime \prime} a b\right) z^{2}=1
$$

und, wenn wir das Quadrat der halben Axenlängen durch $\boldsymbol{r}^{2}$ und den reciproken Werth desselben durch $s^{2}$ bezeichnen,

und hiernach

$$
\frac{1}{s^{2}}=r^{2}=\left(1+a^{2}+b^{2}\right) z^{2}
$$

$$
\text { 5'. } \quad A a^{2}+A^{\prime} b^{2}+A^{\prime \prime}+2 B b+2 B^{\prime} a+2 B^{\prime \prime} a b=\left(1+a^{2}+b^{2}\right) s^{2} .
$$

Addiren wir ferner die Gleichungen (4.), nachdem wir die erste derselben mit $a^{2}$, die zweite mit $b^{2}$ multiplicirt haben, so kommt:

$$
A a^{2}+A^{\prime} b^{2}+B b+B^{\prime} a+2 B^{\prime \prime} a b-\left(B b+B^{\prime} a+A^{\prime \prime}\right)\left(a^{2}+b^{2}\right)=0,
$$

und wenn wir diese Gleichung von der vorhergehenden abziehen und zugleich den Factor $\left(1+a^{2}+b^{2}\right)$ weglassen:

$$
\text { 6. } B^{\prime} a+B b+\left(A^{\prime \prime}-s^{2}\right)=0 \text {. }
$$

Auf gleiche Weise, oder auch durch blofse Buchstabenvertauschung, ergiebt sich:

7. $B^{\prime \prime} a+B+\left(A^{\prime}-s^{2}\right) b=0, \quad B^{\prime}+B^{\prime \prime} b+\left(A-s^{2}\right) a=0$. Endlich erhalten wir, wenn wir $a$ und $b$ zwischen den drei letzten 
Gleichungen eliminiren:
8. $\left(A-s^{2}\right)\left(A^{\prime}-s^{2}\right)\left(A^{\prime \prime}-s^{2}\right)-B^{2}\left(A-s^{2}\right)-B^{\prime 2}\left(A^{\prime}-s^{2}\right)-B^{\prime \prime 2}\left(A^{\prime \prime}-s^{2}\right)$ $+2 B B^{\prime} B^{\prime \prime}=0$

oder auch, wenn wir in Beziehung auf $s^{2}$ orduen:

$$
\text { 9. } \begin{aligned}
s^{6}- & \left(\boldsymbol{A}+\boldsymbol{A}^{\prime}+\boldsymbol{A}^{\prime \prime}\right) s^{4}+\left[\left(\boldsymbol{A} \boldsymbol{A}^{\prime}-\boldsymbol{B}^{\prime \prime 2}\right)+\left(\boldsymbol{A} \boldsymbol{A}^{\prime \prime}-\boldsymbol{B}^{\prime 2}\right)+\left(\boldsymbol{A}^{\prime} \boldsymbol{A}^{\prime \prime}-\boldsymbol{B}^{2}\right)\right] s^{2} \\
- & {\left[\boldsymbol{A} \boldsymbol{A}^{\prime} \boldsymbol{A}^{\prime \prime}-\boldsymbol{A} \boldsymbol{B}^{2}-\boldsymbol{A}^{\prime} \boldsymbol{B}^{\prime 2}-\boldsymbol{A}^{\prime \prime} \boldsymbol{B}^{\prime \prime 2}+2 \boldsymbol{B} \boldsymbol{B}^{\prime} \boldsymbol{B}^{\prime \prime}\right]=0 . }
\end{aligned}
$$

Diese Gleichung giebt drei Werthe für $s^{2}$, den drei Werthenpaaren von $a$ und $b$ entsprechend, welche, wie diese, in Folge der Gleichungen (6.) und (7.) alle drei reell sind. Hiernach bestimmen die Zeichen der Werthe der drei Coëficienten dieser Gleichung unmittelbar, ob die Werthe von $s^{2}$ alle drei positiv, oder alle drei negativ, oder zwei positiv und einer negativ, oder endlich einer positiv und zwei negativ sind. Diesem entsprechend ist dann die Fläche ein Ellipsoïd, eine imaginäre Fläche, ein einschaaliges, oder ein zweischaaliges Hyperboloïd.

4. Das létzte, von $s^{2}$ unabhängige Glied in der Gleichung (9.) ist dem reciproken Werthe des Productes der Quadrate der drei halben Axen der Fläche gleich. Ist diese ein Cylinder, so verschwindet dieses Glied, und dann hat die Gleichung

$s^{4}-\left(A+A^{\prime}+A^{\prime \prime}\right) s^{2}+\left[\left(A A^{\prime}-B^{\prime \prime 2}\right)+\left(A A^{\prime \prime}-B^{\prime 2}\right)+\left(A^{\prime} A^{\prime \prime}-B^{2}\right)\right]=0$ die reciproken Werthe der Quadrate der halben Axen derjenigen Schnitte, welche senkrecht gegen seine Seiten geführt werden, zu ihren Wurzeln. Steht der Cylinder auf einer der drei Coordinaten-Ebenen, etwa auf der Ebene $x y$ senkrecht, so verschwinden aus der allgemeinen Gleichung die drei Coëfficienten $A^{\prime \prime}, B$ und $B^{\prime}$, und die letzte Gleichung reducirt sich auf folgende:

$$
\text { 10. } s^{4}-\left(\boldsymbol{A}+\boldsymbol{A}^{\prime}\right) s^{2}+\left(\boldsymbol{A} \boldsymbol{A}^{\prime}-\boldsymbol{B}^{\prime \prime 2}\right)=0 \text {, }
$$

welche nun noch die reciproken Werthe der beiden halben Axen der Basis des Cylinders, oder, was dasselbe ist, der durch die Gleichung

$$
\boldsymbol{A} \boldsymbol{x}^{2}+\boldsymbol{A} \boldsymbol{y}^{2}+2 \dot{B}^{\prime \prime} \boldsymbol{x y}=1
$$

bei Abstraction von der dritten Dimension dargestellten Curve zweiter Ordnung, zu Wurzeln hat. Für dieselbe Curve ist die Axenrichtung durch die Gleichung (5.) gegeben.

5. Wenn wir derselben Fläche nach einander verschiedene Lagen gegen die Coordinaten-Axen geben, indem wir diese um ihren Anfangspunct irgendwo drehen, so erbalten die Coëfficienten ihrer Gleichung (1.) 
verschiedene Werthe, während die Gleichung (9.) unverändert dieselbe bleibt. $\boldsymbol{A}, \boldsymbol{A}^{\prime}, \boldsymbol{A}^{\prime \prime}$ bedeuten die Quadrate der reciproken Werthe der jedesmaligen Halbdurchmesser, welche in die drei Coordinaten-Axen fallen. Ihre Summe ist also constant.

Wenn man ein System dreier auf einander senkrechten, im Mittelpuncte einer Fläche zweiter Ordnung sich schneidenden geraden Linien um den Mittelpunct beliebig sich drehen lä/st, so ist für alle Lagen die Quadratsumme der reciproken Werthe der in die jedesmäligen drei geraden Linien fallenden Halbdurchmesser der Fläche dieselbe.

Nach der Gleichung (10.) bedeutet $\left(\boldsymbol{A}^{\prime}-B^{\prime \prime 2}\right)$ den reciproken Werth des Products der Quadrate der beiden halben Axen der Durchschnittscurve, also (für den Fall des Ellipsoïds), wenn wir noch durch $\pi^{2}$ dividiren, den reciproken Werth des Quadrats der Fläche dieser Curve. Aehnliche Bedeutung haben die Ausdrücke $\left(\boldsymbol{A} A^{\prime \prime}-B^{\prime 2}\right)$ und $\left(A^{\prime} A^{\prime \prime}-B^{2}\right)$, und sonit giebt die Betrachtung des zweiten Coëfficienten in der Gleichung (9.) den folgenden Satz.

Wenn man ein System dreier auf einander senkrechter und im Mittelpuncte eines Ellipsoz̈ds sich schneidenden Ebenen um diesen Mittelpunct beliebig sich drehen lïfst, so ist für alle Lagen die Quadratsumme der reciproken Werthe des Flächen-Inhalts der drei Durchschnittscurven constant.

Für den Fall eines geraden Cylinders können wir für die drei schneidenden Ebenen irgend drei auf einander senkrecht stehende nehmen; die constante Summe ist alsdann dem Quadrate des reciproken Werthes der Fläche seiner Basis gleich.

\section{2.}

6. Mit derselben Leichtigkeit, wie wir eine Fläche zweiter Ordnung durch ihre Puncte bestimmen, indem wir sie auf gewöhnliche Weise durch eime Gleichung ausdrücken, können wir dieselbe auch durch die sie berührenden Ebenen bestimmen, indem wir sie durch die folgende Gleichung ausdrücken :

$$
\text { 1. } \quad \boldsymbol{A} t^{2}+\boldsymbol{A}^{\prime} u^{2}+\boldsymbol{A}^{\prime \prime} v^{2}+2 \boldsymbol{B} u v+2 \boldsymbol{B}^{\prime} t v+2 \boldsymbol{B}^{\prime \prime} t u=w^{2} \text {. *) }
$$

*) Vergl. Note sur ane théoric nouvelle des surfaces. Journ. Bd. IX. pg. 124. Eine Ebene, welche durch die gewöhnliche Gleichung:

$$
t x+u y+v z=w
$$


Diefs ist die allgemeine Gleichung von Flächen, welche einen Mittelpunct haben; und dieser ist hier zum Anfangspuncte der Coordiuaten genommen.

Die Absicht dieses zweiten Paragraphen ist, nach der neuen analytischen Bezeichnungsweise wiederum die Lage und die Gröfse der Axen zu bestimmen; was hier mit noch grö[serer Leichtigkeit geschehen kann.

7. Wir wollen dabei von der Anschauung ausgehen, dafs, wenn wir eine Schnitt-Ebene durch den Mittelpunct legen, der Pol dieser Ebene nach bestimmter Richtung unendlich weit liege, und dafs, wenn diese Richtung auf der Schnitt-Ebene senkrecht steht, dieselbe ein Hauptschnitt der Fläche zweiter Orduung sei.

Die Gleichung der Schnitt-Ebene in gewöhulichen Coordinaten sei

$$
\text { 2. } t^{\prime} x+u^{\prime} y+v^{\prime} z=0 \text {; }
$$

wonach die beiden Gleichungen einer auf derselben im Anfangspuncte senkrechten geraden Linie die folgenden sind:

$$
\text { 3. } \quad x=\frac{t^{\prime}}{v^{\prime}} z, \quad y=\frac{u^{\prime}}{v^{\prime}} z \text {. }
$$

Für die Gleichung des Poles der Schnitt-Ebene, deren Coordinaten $\boldsymbol{t}^{\prime}, \boldsymbol{u}^{\prime}$, $\boldsymbol{v}^{\prime}, 0$ sind, erhalten wir unnittelbar:

4. $\left(\boldsymbol{A} t^{\prime}+\boldsymbol{B}^{\prime \prime} u^{\prime}+B^{\prime} v^{\prime}\right) \boldsymbol{t}+\left(\boldsymbol{B}^{\prime \prime} t^{\prime}+\boldsymbol{A}^{\prime} u^{\prime}+B v^{\prime}\right) \boldsymbol{u}+\left(\boldsymbol{B}^{\prime} t^{\prime}+B u^{\prime}+\boldsymbol{A}^{\prime \prime} v^{\prime}\right) v$

$$
=0 \text { *). }
$$

dargestellt wird, ist durch die vier Constanten $t, u, v, w$, (von denen wir jede beliebige gleich Eins setzen können, und welche wir Coordinaten der Ebene nennen) bestimmt. Ist zwischen diesen vier Coordinaten blofs eine homogene Gleichung gegeben, so entsprechen dieser unendlich viele Ebenen, welche eine bestimmte Fläche umhüllen. Ist die homogene Gleichung blofs vom ersten Grade, und folgende:

$$
x^{\prime} t+y^{\prime} u+z^{\prime} v=w,
$$

so umhüllen alle Ebenen einen Punct, desseñ Coordinaten $x^{\prime}, y^{\prime}$ und $z^{\prime}$ sind. Fehlt $w$, so liegt der Punct in der Richtung

unendlich weit.

$$
x=\frac{x^{\prime}}{z^{\prime}} \cdot z, \quad y=\frac{y^{\prime}}{z^{\prime}} \cdot z,
$$

*) Wenn wir nemlich, der Kürze wegen, die Gleichung (1.) durch $\Omega=0$ darstellen, so ist die Gleichung des Poles einer gegebenen Ebene, deren Coordinaten $t^{\prime}$, $u^{\prime}, v^{\prime}, w^{\prime}$ sind,

$$
\frac{d \Omega}{d t} \cdot t^{\prime}+\frac{d \Omega}{d u} \cdot u^{\prime}+\frac{d \Omega}{d v} \cdot v^{\prime}+\frac{d \Omega}{d w} \cdot w^{\prime}=0:
$$

eine Gleichung, die sich nicht ändert, wenn wir die Constanten $t^{\prime}, u^{\prime}, v^{\prime}$ und $w^{\prime}$ bezüglich mit den Variabeln $t, u, v, w$ vertauschen. Das letzte Glied fält aus, wenn $v^{\prime}=0$ ist.

Crelle's Journal f. d. M. Bd. XXIV. Hft. 1. 
Der Pol liegt unendlich weit, weil $w$ fehlt. Die Richtung, nach welcher er unendlich weit liegt, mufs also mit der Richtung der Linie (3.) zusammenfallen, wenn (2.) ein Hauptschnitt der Fläche sein soll. Diefs geschieht, wenn

5. $\quad \frac{\boldsymbol{A} t^{\prime}+\boldsymbol{B}^{\prime \prime} u^{\prime}+\boldsymbol{B}^{\prime} v^{\prime}}{\boldsymbol{B}^{\prime} t^{\prime}+\boldsymbol{B} u^{\prime}+\boldsymbol{A}^{\prime \prime} v^{\prime}}=\frac{t^{\prime}}{v^{\prime}}, \quad \frac{\boldsymbol{B}^{\prime \prime} t^{\prime}+\boldsymbol{A}^{\prime} u^{\prime}+\boldsymbol{B} v^{\prime}}{\boldsymbol{B}^{\prime} t^{\prime}+\boldsymbol{B} u^{\prime}+\boldsymbol{A}^{\prime \prime} v^{\prime}}=\frac{u^{\prime}}{v^{\prime}}$ ist.

Schaffen wir aus diesen Gleichungen die Nenner fort, und setzen überdies $v^{\prime}=0$, so kommt

$$
\text { 6. } \quad\left\{\begin{aligned}
\boldsymbol{A} t^{\prime}+\boldsymbol{B}^{\prime \prime} u^{\prime}+\boldsymbol{B}^{\prime}-\left(\boldsymbol{B}^{\prime} t^{\prime}+\boldsymbol{B} u^{\prime}+\boldsymbol{A}^{\prime \prime}\right) t^{\prime}=0, \\
\boldsymbol{B}^{\prime \prime} t^{\prime}+\boldsymbol{A}^{\prime} u^{\prime}+\boldsymbol{B}-\left(\boldsymbol{B}^{\prime} t^{\prime}+\boldsymbol{B} u^{\prime}+\boldsymbol{A}^{\prime \prime}\right) \boldsymbol{u}^{\prime}=0 .
\end{aligned}\right.
$$

Durch diese Gleichungen sind drei Werthenpaare für $\boldsymbol{t}^{t}$ und $\boldsymbol{u}^{\prime}$ und dadurch die drei Hauptschnitte der Fläche bestimmt. Diese, und also auch die drei Axen, sind reell, weil die vorstehenden Gleichungen genau dieselben Coëfficienten haben, wie die Gleichungen (4.) der 2. Nummer.

8. Wenn wir, wie in der Note zur vorigen Nummer, die Gleichung der Fläche durch

$$
\Omega=0
$$

darstellen, so können wir die Gleichungen (5.) durch die folgenden ersetzen :

$$
\frac{d \Omega}{d v} v=\frac{d \Omega}{d t} t, \quad \frac{d \Omega}{d v} v=\frac{d \Omega}{d u} u .
$$

Die durch diese beiden Gleichungen gegebenen Werthe von $\frac{t}{v}$ und $\frac{u}{v}$ bestimmen die Lage der drei Hauptschnitte.

9. Wenn wir in der Gleichung der Fläche

setzen, so ergiebt sich

$$
t=t^{\prime}, \quad u=u^{\prime}, \quad v=1
$$

$$
\boldsymbol{A} \boldsymbol{t}^{\prime 2}+\boldsymbol{A}^{\prime} \boldsymbol{u}^{\prime 2}+\boldsymbol{A}^{\prime \prime}+2 \boldsymbol{B} \boldsymbol{u}^{\prime}+2 \boldsymbol{B}^{\prime} \boldsymbol{t}^{\prime}+2 \boldsymbol{B}^{\prime \prime} t u=w^{2}
$$

wo alsdann $w$ dasjenige Segment bedeutet, welches eine mit einem Hauptschnitte parallele Tangential-Ebene der Fläche von der Axe der $z$ abschneidet. Der senkrechte Abstand des Anfangspunctes der Coordinaten von dieser Tangential-Ebene ist eine halbe Axe der Fläche. Bezeichnen wir die Länge derselben durch $r$, so ergiebt sich

und mithin

$$
w^{2}=\left(1+t^{\prime 2}+u^{\prime 2}\right) r^{2}
$$

$$
\boldsymbol{A} \boldsymbol{t}^{\prime 2}+\boldsymbol{A}^{\prime} \boldsymbol{u}^{\prime 2}+\boldsymbol{A}^{\prime \prime}+2 \boldsymbol{B} \boldsymbol{u}^{\prime}+2 \boldsymbol{B}^{\prime} \boldsymbol{t}^{\prime}+2 \boldsymbol{B}^{\prime \prime} \boldsymbol{t}^{\prime} \boldsymbol{u}^{\prime}=\left(1+\boldsymbol{t}^{\prime 2}+\boldsymbol{u}^{\prime 2}\right) \boldsymbol{r}^{2}
$$

Diese Gleichung hat wiederum genau dieselbe Form, wie die Gleichung (5') der 3. Nummer. Wenn wir sie daher mit den Gleichungen (6.) zusammen- 
stellen, so erhalten wir die folgenden Gleichungen:

$$
\text { 7. }\left\{\begin{aligned}
B^{\prime} t^{\prime}+B u^{\prime}+\left(A^{\prime \prime}-r^{2}\right)=0 \\
B^{\prime \prime} t^{\prime}+B+\left(A^{\prime}-r^{2}\right) u^{\prime}=0 \\
B^{\prime}+B^{\prime \prime} u^{\prime}+\left(A-r^{2}\right) t^{\prime}=0
\end{aligned}\right.
$$

8. $\left(A-r^{2}\right)\left(A^{\prime}-r^{2}\right)\left(A^{\prime \prime}-r^{2}\right)-B^{2}\left(A-r^{2}\right)-B^{\prime 2}\left(A^{\prime}-r^{2}\right)-B^{\prime 2}\left(A^{\prime \prime}-r^{2}\right)$ $+2 \boldsymbol{B} \boldsymbol{B}^{\prime} \boldsymbol{B}^{\prime \prime}=0$

9. $\left.\boldsymbol{r}^{6}-\left(\boldsymbol{A}+\boldsymbol{A}^{\prime}+\boldsymbol{A}^{\prime \prime}\right) \boldsymbol{r}^{4}+\left[\left(\boldsymbol{A} \boldsymbol{A}^{\prime}-\boldsymbol{B}^{\prime \prime 2}\right)+\left(\boldsymbol{A} \boldsymbol{A}^{\prime \prime}-\boldsymbol{B}^{\prime 2}\right)+\boldsymbol{A}^{\prime} \boldsymbol{A}^{\prime \prime}-\boldsymbol{B}^{2}\right)\right] \boldsymbol{r}^{2}$ $-\left[\boldsymbol{A} \boldsymbol{A}^{\prime} \boldsymbol{A}^{\prime \prime}-\boldsymbol{A} \boldsymbol{B}^{2}-\boldsymbol{A}^{\prime} \boldsymbol{B}^{\prime 2}-\boldsymbol{A}^{\prime \prime} \boldsymbol{B}^{\prime \prime 2}+2 \boldsymbol{B} \boldsymbol{B}^{\prime} \boldsymbol{B}^{\prime \prime}\right]=0$,

die wir unmittelbar hinschreiben können, weil auch sie dieselbe Form haben müssen, wie die entsprechenden Gleichungen der 3. Nummer.

Die Gleichungen (7.) können wir, analog wie in der 8. Nummer, indem wir die partiellen Differentialcoëfficienten auf die Coordinaten der Hauptschnitte beziehen, auch in folgender Form schreiben:

$$
s^{2}=\frac{1}{v^{\prime}} \cdot \frac{d \Omega^{\prime}}{d v^{\prime}}=\frac{1}{u^{\prime}} \cdot \frac{d \Omega^{\prime}}{d u^{\prime}}=\frac{1}{t^{\prime}} \cdot \frac{d \Omega^{\prime}}{d t} .
$$

10. An die vorstehende Gleichung (9.) knüpfen sich ähnliche Bemerkungen, wie an die Gleichung (9.) in der 4. Nummer. Die neue Gleichung hat genau dieselben Coëfficienten. Ihre drei Wurzeln sind also reell; ob ihre Werthe positiv oder negativ und demnach die drei Axen der Fläche bezüglich reell oder imaginär sind, hängt von denselben Bedingungen ab, wie früher. Wir erhalten also auch, gleichviel, ob wir die Flächen zweiter Ordnung durch die Gleichnng (1.) der 1. oder durch die Gleichung (1.) der 6. Nummer darstellen, genau dieselben Bedingungen zwischen den Coëfficienten, um die verschiedenen Haupt-Arten,von Flächen dieser Ordnung zu unterscheiden. Wenn insbesondere das constante Glied in der vorstehenden Gleichung (9.) verschwindet, so geht die Fläche, indem eine Axe derselben (die früher bei dieser Voraussetzung unendlich wurde) verșchwindet, in eine ebene Curve über, welche insbesondere in der Ebene der $x y$ liegt, wenn aus der allgemeinen Gleichung die drei Coëfficienten $A^{\prime \prime}, \boldsymbol{B}$ und $\boldsymbol{B}^{\prime}$ ganz verschwinden. Diese Gleichung geht alsdann in die folgende über:

$$
\text { 10. } A t^{2}+A^{\prime} u^{2}+2 B^{\prime \prime} t u=1,
$$

und zur Bestimmung der beiden halben Axen der bezüglichen Curve ergiebt sich

$$
\text { 11. } r^{4}-\left(A+A^{\prime}\right) r^{2}+\left(A A^{\prime}-B^{\prime \prime 2}\right)=\underset{9}{0} \text {. }
$$


7. Plücker, Aphorismen aus der Geometrie des Raumes.

11. Wenn wir der Fläche nach einander verschiedene Lagen geben, so ändern sich die Coëfficienten ihrer Gleichung; diese Aenderung aber muls von der Art sein, dafs die drei Coëfficienten in der Gleichung (9.) unverändert bleiben. Es bedeuten aber $A, A^{\prime}, A^{\prime \prime}$ in der allgemeinen Gleichung die Quadrate derjenigen Perpendikel, welche, vom Mittelpuncte der Fläche aus, auf die drei, den jedesmaligen Coordinaten-Ebenen parallele Berührungs-Ebenen gefällt werden können. Die Summe dieser Quadrate, und also auch die Entfernung des gemeinsamen Durchschnittspunctes der drei Taugential-Ebenen vom Mittelpuncte, ist constant.

Der geometrische Ort für körperliche Ecken, u'elche von drei beliebigen, paarweise auf einander senkrechten und eine gegebene Fläche (und insbesondere auch Curve) zweiter Ordnung berührenden Ebenen gebildet werden, ist eine Kugel, für welche das Quadrat des Radius der Quadratsumme der drei halben Axen gleich ist.

Es bedeutet ferner, in Folge der Gleichungen (11.), der Ausdruck $\left(\boldsymbol{A} \boldsymbol{A}^{\prime}-\boldsymbol{B}^{\prime \prime 2}\right)$ das Product der Quadrate der beiden halben Axen der Curve (10.), welche die Basis des der Fläche umschriebenen, auf der Ebene $x y$ senkrechten Cylinders, oder, was dasselbe ist, die Projection des Ellipsoïds auf diese Ebene ist. Das Quadrat dieser Projection wird also durch $\pi^{2}\left(\boldsymbol{A} A^{\prime}-B^{\prime \prime 2}\right)$ dargestellt. Eben so bedeuten $\pi^{2}\left(A A^{\prime \prime}-B^{\prime 2}\right)$ und $\pi^{\prime}\left(\boldsymbol{A}^{\prime} \boldsymbol{A}^{\prime \prime}-\boldsymbol{B}^{2}\right)$ die Projectionen des Ellipsoïds auf die Ebenen $x \boldsymbol{z}$ und $y z$. Hiernach ergiebt sich, wenn wir den Coëfficienten von $\boldsymbol{r}^{2}$ in der Gleichung (9.) betrachten, der folgende Satz:

Die Quadratsumme der drei Projectionsflüchen eines gegebenen Ellipsoüds auf irgend drei auf einander senkrechte Ebenen ist constant.

Bonn am 29sten März 1842.

(Die Fortsetzung folgt.) 